\title{
How community ecology can improve our understanding of cholera dynamics
}

\author{
Guillaume Constantin de Magny ${ }^{1}{ }^{*}$, Nur A. Hasan ${ }^{2}$ and Benjamin Roche ${ }^{3}$ \\ ' Maladies Infectieuses et Vecteurs Ecologie, Génétique, Evolution et Contrôle, Institut de Recherche pour le Développement, UMR 224 IRD-5290 \\ CNRS-UM1-UM2, Montpellier, France \\ ${ }^{2}$ Maryland Pathogen Research Institute, University of Maryland, College Park, MD, USA \\ ${ }^{3}$ Unité de Modélisation Mathématique et Informatique des Systèmes Complexes, Institut de Recherche pour le Developpement, UMI IRD/UPMC 209, \\ Bondy, France
}

\author{
Edited by: \\ Daniela Ceccarelli, University of \\ Maryland, USA

\section{Reviewed by:} \\ Ryan J. Newton, University of \\ Wisconsin-Milwaukee, USA \\ Yan Boucher, University of Alberta, \\ Canada

\section{${ }^{*}$ Correspondence:} \\ Guillaume Constantin de Magny, \\ Maladies Infectieuses et Vecteurs \\ Ecologie, Génétique, Evolution et \\ Contrôle, Institut de Recherche pour \\ le Développement, UMR 224 \\ IRD-5290 CNRS-UM1-UM2, 911 \\ avenue Agropolis, BP 64501 \\ Montpellier, France \\ e-mail: guillaume.demagny@ird.fr
}

Understanding the seasonal emergence and reemergence of cholera is challenging due to the complex dynamics of different protagonists. The abundance of Vibrio cholerae, the causative agent of cholera and a natural inhabitant of aquatic environments, fluctuates according to abiotic, and biotic factors. Among the biotic factors, the zooplankton community dynamics has been suggested to play a pivotal role in the survival, persistence, and natural competence of $V$. cholerae. However, factors regulating $V$. cholerae population structure and seasonal dynamics are still not fully understood. Investigation of the temporal shifts and variability in aquatic community composition in relation to the occurrence or abundance of $V$. cholerae appears very promising yet remained underexplored. Recent advances in metagenomics, facilitated by high-throughput ultra deep sequencing, have greatly improved our ability for a broader and deeper exploration of microbial communities including an understanding of community structure, function, as well as inter- and intra-specific competitions. Here, we discuss possible areas of research focusing how combination of community ecology and metagenomic approaches could be applied to study the cholera system.
The diarrheal disease, cholera, is caused by the pathogenic bacteria Vibrio cholerae; affecting hundreds of thousands of people every year with thousands of deaths (World Health Organization, 2010). Depending on the health status, infection occurs due to the ingestion of $10^{3}-10^{9}$ cells of pathogenic $V$. cholerae (either O1 or O139) through contaminated food and water. After the ingestion, the microbe has to pass through acidic conditions of the human stomach and the immune system defenses to reach the small intestine, where it attaches, and begins to produce cholera toxin (CT). Sufficient quantities of CT cause severe diarrhea and shedding of the pathogen, up to $10^{7}$ per infected individual per stool (Zahid et al., 2008), into the environment facilitating spread of the disease.

Outside the human host, V. cholerae is an autochthonous member of natural aquatic environments such as lakes, rivers, estuaries, and the ocean, which serve as the principal natural reservoir for this organism. Ecologically, vibrios play an important role in the degradation of organic matter and act as a link that transfers dissolved organic carbon to higher trophic levels of the marine food web (Grossart et al., 2005). V. cholerae is a facultative anaerobic, asporogenous, gram-negative rod with capability of respiratory and fermentative metabolism. It is oxidase positive, reduces nitrate, and motile by a single polar, sheathed flagellum (Kaper et al., 1995). The pathogenic and non-pathogenic strains of $V$. cholerae are globally distributed in aquatic environments (Lipp et al., 2002). Historically, cholera is endemic in the Bengal basin with outbreaks occurring elsewhere due to lack of access to clean water and sanitation facilities (World Health Organization, 2010). The role of climate and other abiotic factors on cholera has been well investigated (Lipp et al., 2002) but approaches taken to date to study $V$. cholerae have fallen short in separating the role of abiotic and biotic factors. Therefore, we are advocating the need for both genomics and community ecology approaches to elucidate such relationships.

How $V$. cholerae survives in two very distinct habitats, human hosts, and aquatic ecosystems, and mechanisms by which it periodically emerges as a human pathogen are compelling questions in the ecology of this species. Understanding the ecology of $V$. cholerae is, however, challenging because of the complex dynamics of different protagonists. In this paper, we will explore how knowledge from multiple different disciplines like ecology, genomics, and modeling, might contribute to establish an ecological framework of $V$. cholerae to provide critical insights in our understanding of this bacterium.

A widely accepted concept in theoretical ecology of V. cholerae is the niche theory. The niche theory is defined by three main factors that contribute to population growth rate: resources, the physical environment, and natural enemies (Vandermeer, 1972). How a species respond to these factors, spatially, and temporarily, determines its ability to invade, persist, and occasionally become dominant. This appears as a critical phenomenon in understanding the ecology of $V$. cholerae.

The first factor is resources. For V. cholerae, this factor is strongly linked to the availability of chitin in the natural environment. In 
the aquatic ecosystem, chitin is the most abundant polysaccharide and the principal component of zooplankton exoskeleton. $V$. cholerae is strongly associated with plankton, forming commensal relationships with chitinous organisms that are dominant among zooplankton populations (i.e., copepods, amphipods, and other crustaceans; Colwell and Huq, 1994). The copepod exoskeleton has been shown to support large population of vibrios, including the pathogenic V. cholerae (Colwell et al., 1981; Huq et al., 1983; Tamplin et al., 1990). An advantage of epibiotic organisms, such as $V$. cholerae, on biotic substrates, is their proximity to available nutrients. V. cholerae cells living on highly mobile zooplanktons are less likely to be nutrient limited than "free-living" or planktonic cells in the environment.

The commensal relationship between vibrios and chitinous organisms, like copepods, has important consequences (Pruzzo etal., 2008). This relationship represents a useful model to investigate the role of primary habitat selection in developing pathogenicity traits of the bacteria primarily inhabiting the aquatic environment. It has also been reported that $V$. cholerae becomes naturally competent and amenable to serotype conversion on chitin surfaces (Meibom et al., 2005). Hence, the ability of chitin to support both genetic evolutionary diversity and growth of cholera organisms in the environment strongly implicates copepods as a critical component in the environmental lifestyle of this human pathogen. Indeed, zooplanktons comprise a broad assortment of ecologically important heterotrophic groups. The composition of zooplankton community changes constantly during an annual cycle, with dramatic differences in species composition of freshwater and marine zooplankton communities. Thus, the plankton species composition plays a pivotal role in Vibrio seasonality, as seen, i.e., in waters off the Georgia (U.S.) coast, highlighting the complex relationship between seasonal shifts in plankton composition and number of vibrios in the aquatic environment (Turner et al., 2009).

Statistical model parameter estimates have indicated a strong direct relationship between Vibrio concentration and the relative abundance of copepods in the $>200 \mu \mathrm{m}$ fraction. Recently, the incidence of cholera and the occurrence of pathogenic V. cholerae with diverse zooplankton taxa were studied in rural areas of Bangladesh (Constantin de Magny et al., 2011). Chitinous zooplankton communities of several water bodies were analyzed in order to understand the interaction of the zooplankton population composition with the population dynamics of pathogenic $V$. cholerae and the incidence of cholera. Two dominant zooplankton groups, namely, rotifers and cladocerans, were consistently associated with detection of $V$. cholerae and/or occurrence of cholera cases. Specifically, the presence of rotifer, Brachionus angularis, was significantly associated with the presence of pathogenic $V$. cholerae. Local differences indicated some subtle ecological factors influencing the interactions between $V$. cholerae, its plankton hosts, and incidence of cholera. Like the determination of a potential keystone species (Mills and Doak, 1993), or assemblage of species associated with the occurrence of $V$. cholerae, understanding the community dynamics (diversity and abundance) of zooplanktons in the natural aquatic environment could be a key element in deciphering the complete picture of $V$. cholerae ecology.
The second factor of the niche theory for V. cholerae is the physical environment. For most Vibrio sp., temperature and salinity are two major abiotic factors strongly associated with their abundance (Lipp et al., 2002). Materna et al. (2012) remarkably investigated the shape and evolution of the fundamental niche of marine Vibrio by combining theoretical and experimental approaches on 2-dimensional niche shape associated with temperature and salinity, the two major factors in Vibrio ecology. It was found that for the studied marine Vibrio strains, ecological range, in reality, occupies a limited section of their potential niche (Materna et al., 2012). In addition to the physical drivers of the population growth, this bacterial species, like other gram-negative bacteria, has a selective advantage in its ability to enter a dormant stage when environmental conditions are unfavorable for active growth and cell division. This state is termed the viable but nonculturable (VBNC) state (Cottingham et al., 2003; Takeda, 2011). This represents another important aspect of the ecological niche of $V$. cholerae, namely its adaptability to fluctuations of the abiotic conditions.

The VBNC state of bacteria is defined as the state where cells remain viable but cease to grow or divide, on, or in, routinely used bacteriological media. Even if it is still in debate, evidences of the reversibility of this state back to active growth and cell division have accumulated (Senoh et al., 2010; Takeda, 2011). This nonculturability associated with the ability to aggregate on biofilm, even after having been non-culturable for more than one year (Alam et al., 2007), is another important aspect in the ecology of $V$. cholerae. Several studies have already demonstrated the importance of the VBNC state in the seasonal epidemics of cholera (Colwell et al., 1985, 1996; Alam et al., 2006a,b; Alam et al., 2007; Asakura et al., 2007). A recent study (Mishra et al., 2012), however, demonstrated a vital role of the VBNC state in cholera epidemiology manifesting an active expression of traits necessary for both viability, stress response, virulence, and colonization of the bacterium.

Yet another factor of the ecological niche of $V$. cholerae is the biotic antagonists. Among them, the lysogenic filamentous phage, СТХ $\Phi$, is found in toxigenic V. cholerae (Waldor and Mekalanos, 1996) and encodes the CT. The V. cholerae pathogenicity island, VPI, another antagonist, encodes a toxin co-regulated pilus that functions as a colonization factor, and a receptor for СТХ $\Phi$. It has been hypothesized that lytic bacterial viruses are responsible for the decline in the incidence of cholera during seasonal outbreaks (Faruque et al., 2005b), limiting V. cholerae population densities through predation. It has been suggested that the increased environmental phage abundance is the result of a host-mediated phage amplification during the cholera epidemic, decreasing the load of environmental $V$. cholerae resulting in the collapse of the epidemic (Faruque etal., 2005a). This predator/prey relationship have been debated due to the lack of evidences distinguishing the effect of growth-limiting resources and the lytic activity of the phage restricting the densities of V. cholerae population (Wei et al., 2010). The alternative hypothesis states that phage-resistant $V$. cholerae mutants are less fit than wild-type, possessing a number of characteristics that are likely to further reduce capacity to be maintained in natural populations and may be avirulent in human hosts (Wei et al., 2010). The role 
of the combined effect of growth resources and the lytic activity of phages on $V$. cholerae population abundances need to be clarified for better understanding of bacterial population dynamics driver.

In addition, it appears that $V$. cholerae does not give up without a fight. V. cholerae, like other Gram-negative bacteria, utilizes accessory virulence factors, e.g., the type VI secretion system (T6SS), to provide advantages in intraspecific and interspecific competition (Unterweger et al., 2012). It is hypothesized that the T6SS and toxin translocation play critical roles for $V$. cholerae to outcompete other bacteria and phagocytic cells. Thus, it persists in human hosts and in the environment (Miyata et al., 2010). Furthermore, it was shown that in order to successfully colonize organic matter in the marine environment, $V$. cholerae must compete against a high concentration of diverse bacteria that reside on the surface of particles. The interspecies antagonistic interactions involving allelochemicals can influence $V$. cholerae particle colonizations. These interactions can also be temperature sensitive (Long et al., 2005). However, the definite role of the microbial (viral and bacterial) community on $V$. cholerae abundance is still unknown. As microbes are interacting with each other, at an individual scale (Hajishengallis et al., 2012), or at a population level (Telfer et al., 2010), overlooking such interaction may lead to a partial understanding of the contributing factors. However, microbial communities are typically very diverse and complex, therefore, characterization of species diversity and the complex network of species interactions within these communities also pose a technical challenge to the scientific community.

The advent of next-generation sequencing technologies and bioinformatics opened up the door to a wide new era of research exploration and has offered an unprecedented opportunity to fill an existing knowledge gap in community ecology. Recent advances of such technologies and tools have already revolutionized the field of metagenomics, allowing a much more accurate, and detailed description of microbial communities. As these technologies and tools are becoming widely available and accessible, their application is becoming the cornerstone in addressing the outstanding hypotheses in various domains like biogeography as well as furthering our understanding of how ecological communities assemble, evolve, and function. Next-generation high-throughput sequencing (HTS) methods have already been proven as an outstanding way to describe microbial biodiversity and community ecology in a variety of systems and environments. By definition, an individual ecosystem is composed of populations of organisms, interacting within communities, and contributing to the cycling of nutrients, and the flow of energy. Conventional methods of sampling ecosystems are unquestionably biased toward the highly abundant and easy to characterize members of the community where HTS methods facilitate the opportunity to study every member of the community either individually, or as a whole, to reflect the entire microbial system. Additionally, decomposition and nutrient cycling are key processes in ecosystem functioning. Both are fundamental to ecosystem biomass production, and mostly operated by microbes, more precisely bacteria. Understanding microbial community dynamics in ecosystems is, therefore, one of the key elements deciphering ecosystem functioning. As $V$. cholerae survives in two very distinct habitats, human hosts, and aquatic ecosystems, it is, therefore, critical to investigate the influence of microbial population dynamics on seasonal emergence and reemergence of $V$. cholerae, especially to understand what happens in the community when there is an outbreak.

Historically, the first metagenomics research was focused on cultured soil microorganisms (Handelsman et al., 1998). Rapidly, it became a very promising source of knowledge in understanding the dynamics of microbial population, the interaction between pathogens, and the microbial community, and how they evolve in different habitats (e.g., host, vector, reservoir). Recently, Monira et al. (2013) explored gut microbiota of children suffering from cholera and during disease recovery. From the nine children included in the study, they reported $V$. cholerae accounted for 35\% (minimum 5\%, maximum 63\%) of the total gut microbiota at day 0 , with $V$. cholerae was the predominant species in six of the nine children (Monira et al., 2013). During the recovery, the effect of antibiotic therapy demonstrated a rapid shift in bacterial diversity affecting the top ten bacterial families, which accounted for more than $85 \%$ of the bacterial flora during cholera. Furthermore, cholera infection appears to induce expulsion of major commensal bacteria belonging to the phyla, e.g., Bacteroidetes, Firmicutes, and Actinobacteria, facilitating the colonization of the gut by harmful bacteria belonging to the phylum Proteobacteria (Monira et al., 2013).

Does this phenomenon of interspecific competition observed in the human gut during cholera infection also occur in the second distinct habitat, the aquatic ecosystem? Other compelling questions in microbial community ecology also arise from this. What is the structure and composition of the microbial communities in the aquatic environment directly sourced by the human populations for their daily needs? How did these microbial communities evolve over time? What space does $V$. cholerae occupy in the communities and how does it impact the cholera dynamics (emergence, transmission)? How similar microbial communities are in the aquatic environments where cholera is endemic, epidemic, or benign? Ongoing research in Bangladesh based on the limited scale application of 16S rRNA gene survey suggests that the abundance and diversity of the microbial community including Vibrio populations exhibited a complex seasonal distribution driven by temporal fluctuations of environmental parameters, such as sea surface temperature, and indicates that a specific consortium among the indigenous population, and their interaction might be crucial in responding to changes in environmental parameters that allow $V$. cholerae to overcome the natural competition and emerge in epidemic proportions.

Translating these capacities of a metagenomics approach to the study of $V$. cholerae, we suggest that such approach could provide crucial insights to infer competition processes within microbial communities. While studies thus far have focused mostly on the environmental dimension of $V$. cholerae niche (Preheim et al., 2011a,b; Materna et al., 2012), adopting metagenomics based community ecology approach could add a critical, perhaps keystone, dimension to the study of $V$. cholerae, and the robustness of predictive models could improve substantially by incorporating such a fundamental dimension. 
Potentially, the integration of all the knowledge about the disease cholera, and the ecology of $V$. cholerae, should lead to an End-To-End model of cholera. End-to-End models are integrated models of an ecosystem, which encompasses climate, various ecosystem components, and in some cases human interaction with the ecosystem. These models are developed to describe, understand, and predict ecosystem dynamics. To this extent, integrating an ecosystem component in such a model, and the microbial community context involving natural enemies of bacteria that influence strongly the kinetics of $V$. cholerae in the environment and, consequently, in human populations, would be crucial in achieving the objective of such a predictive tool. This is in contrast to the traditional approach or the old paradigm of climate prediction for human health that seeks correlations between climatic variables, morbidity, mortality, exacerbation of chronic conditions, population-level outbreaks, or other indicators that are precursors to an outbreak. Additionally, there is a critical need to plug ecological niche modeling (ENM) into this approach. ENM is often used to inform conservation efforts (Peterson and Robins, 2003), to describe species potential range (Meentemeyer et al., 2004), and is increasingly being used to identify species of public health concern (Mullins et al., 2011). Advances so far in environmental predictions for human health can be illustrated by the excellent analogy for Earth System interactions provided by El Niño. Examples range from heat and cold wave related mortalities and morbidities to cholera, malaria, dengue, and meningitis. The impacts of global changes are already manifesting themselves in global indicators such as temperature and sea level rise, but the impacts on populations occur through local changes in weather, ecology, water resources, barometric pressure, etc.

The salience, reliability, and legitimacy of a successful prediction system will depend on filling the gaps in mechanistic linkages from changes in climate to effects on human health and well-being. Thus, simple empirical approaches of the past must be steered toward more mechanistic methodologies to identify the drivers from all fronts, e.g., environment, agriculture, and water. These include genetic, chemical, and biological factors, such as microbial contamination pathways, human behavior, exposure, social, and health systems infrastructure, demographics, and other measures of vulnerability that affect the onset, spread, and exacerbation of disease, and the resources needed (water, food, shelter) to maintain health. Networked phenomena including the flow and potency of pathogens will be a useful component. In addition to geographic measures, vulnerability, and risk clearly depend on various socio-economic and demographic factors. Regional Earth System Prediction can account for these factors in an adaptive, learningby-doing mode of model-data synthesis. More importantly, these disparate pieces have to be integrated into Earth System models, especially in the high resolution RESP framework (Murtugudde, 2009) with goal-oriented observational monitoring networks.

\section{REFERENCES}

Alam, M., Hasan, N. A., Sadique, A., Bhuiyan, N. A., Ahmed, K. U., Nusrin, S., et al. (2006a). Seasonal cholera caused by Vibrio cholerae serogroups O1 and O139 in the coastal aquatic environment of Bangladesh. Appl. Environ. Microbiol. 72, 4096-4104. doi: 10.1128/AEM.00066-06
Alam, M., Sultana, M., Nair, G. B., Sack, R. B., Sack, D. A., Siddique, A. K., et al. (2006b). Toxigenic Vibrio cholerae in the aquatic environment of Mathbaria, Bangladesh. Appl. Environ. Microbiol. 72, 2849-2855. doi: 10.1128/AEM.72.4.2849-2855.2006

Alam, M., Sultana, M., Nair, G. B., Siddique, A. K., Hasan, N. A., Sack, R. B., et al. (2007). Viable but non-culturable Vibrio cholerae O1 in biofilms in the aquatic environment and their role in cholera transmission. Proc. Natl. Acad. Sci. U.S.A. 104, 17801-17806. doi: 10.1073/pnas.0705599104

Asakura, H., Ishiwa, A., Arakawa, E., Makino, S., Okada, Y., Yamamoto, S., et al. (2007). Gene expression profile of Vibrio cholerae in the cold stress-induced viable but non-culturable state. Environ. Microbiol. 9, 869-879. doi: 10.1111/j.14622920.2006.01206.x

Colwell, R. R., Brayton, P., Herrington, D., Tall, B., Huq, A., and Levine, M. M. (1996). Viable but non-culturable Vibrio cholerae O1 revert to a cultivable state in the human intestine. World J. Microbiol. Biotechnol. 12, 28-31. doi: 10.1007/BF00327795

Colwell, R. R., Brayton, P. R., Grimes, D. J., Roszak, D.-R., Huq, A., and Palmer, L. M. (1985). Viable, but non-culturable Vibrio cholerae and related pathogens in the environment: implication for release of genetically engineered microorganisms. Biotechnology 3, 817-820. doi: 10.1038/nbt0985-817

Colwell, R. R., and Huq, A. (1994). Environmental reservoir of Vibrio cholerae. The causative agent of cholera. Ann. N. Y. Acad. Sci. 740, 44-54. doi: 10.1111/j.17496632.1994.tb19852.x

Colwell, R. R., Seidler, R. J., Kaper, J., Joseph, S. W., Garges, S., Lockman, H., et al. (1981). Occurrence of Vibrio cholerae serotype O1 in Maryland and Louisiana estuaries. Appl. Environ. Microbiol. 41, 555-558.

Constantin de Magny, G., Mozumder, P. K., Grim, C. J., Hasan, N. A., Naser, M. N., Alam, M., etal. (2011). Role of zooplankton diversity in Vibrio cholerae population dynamics and in the incidence of cholera in the Bangladesh Sundarbans. Appl. Environ. Microbiol. 77, 6125-6132. doi: 10.1128/AEM. 01472-10

Cottingham, K. L., Chiavelli, D. A., and Taylor, R. K. (2003). Environmental microbe and human pathogen: the ecology and microbiology of Vibrio cholerae. Front. Ecol. Environ. 1:80-86. doi: 10.1890/15409295(2003)001[0080:EMAHPT]2.0.CO;2

Faruque, S. M., Islam, M. J., Ahmad, Q. S., Faruque, A. S. G., Sack, D. A., Nair, G. B., et al. (2005a). Self-limiting nature of seasonal cholera epidemics: role of hostmediated amplification of phage. Proc. Natl. Acad. Sci. U.S.A. 102, 6119-6124. doi: 10.1073/pnas.0502069102

Faruque, S. M., Naser, I. B., Islam, M. J., Faruque, A. S., Ghosh, A. N., Nair, G. B., et al. (2005b). Seasonal epidemics of cholera inversely correlate with the prevalence of environmental cholera phages. Proc. Natl. Acad. Sci. U.S.A.102, 1702-1707. doi: 10.1073/pnas.0408992102

Grossart, H.-P., Levold, F., Allgaier, M., Simon, M., and Brinkhoff, T. (2005). Marine diatom species harbor distinct bacterial communities. Environ. Microbiol. 7, 860873. doi: 10.1111/j.1462-2920.2005.00759.x

Hajishengallis, G., Darveau, R. P., and Curtis, M. A. (2012). The keystonepathogen hypothesis. Nat. Rev. Microbiol. 10, 717-725. doi: 10.1038/nrmicro 2873

Handelsman, J., Rondon, M. R., Brady, S. F., Clardy, J., and Goodman, R. M. (1998). Molecular biological access to the chemistry of unknown soil microbes: a new frontier for natural products. Chem. Biol. 5, R245-R249. doi: 10.1016/S10745521(98)90108-9

Huq, A., Small, E. B., West, P. A., Huq, M. I., Rahman, R., and Colwell, R. R. (1983). Ecological relationships between Vibrio cholerae and planktonic crustacean copepods. Appl. Env. Microbiol. 45, 275-283.

Kaper, J. B., Morris, J. G. Jr., and Levine, M. M. (1995). Cholera. Clin. Microbiol. Rev. 8, 48-86.

Lipp, E. K., Huq, A., and Colwell, R. R. (2002). Effects of global climate on infectious disease: the cholera model. Clin. Microbiol. Rev. 15, 757-770. doi: 10.1128/CMR.15.4.757-770.2002

Long, R. A., Rowley, D. C., Zamora, E., Liu, J., Bartlett, D. H., and Azam, F. (2005). Antagonistic interactions among marine bacteria impede the proliferation of Vibrio cholerae. Appl. Environ. Microbiol. 71, 8531-8536. doi: 10.1128/AEM.71.12.8531-8536.2005

Materna, A. C., Friedman, J., Bauer, C., David, C., Chen, S., Huang, I. B., et al. (2012). Shape and evolution of the fundamental niche in marine Vibrio. ISME J. 6, 2168-2177. doi: 10.1038/ismej.2012.65 
Meentemeyer, R., Rizzo, D., Mark, W., and Lotz, E. (2004). Mapping the risk of establishment and spread of sudden oak death in California. For. Ecol. Manage. 200, 195-214. doi: 10.1016/j.foreco.2004.06.021

Meibom, K. L., Blokesch, M., Dolganov, N. A., Wu, C.-Y., and Schoolnik, G. K. (2005). Chitin induces natural competence in Vibrio cholerae. Science 310, 1824 1827. doi: 10.1126/science.1120096

Mills, L. S., and Doak, D. F. (1993). The keystone-species concept in ecology and conservation. Bioscience 43, 219-224. doi: 10.2307/1312122

Mishra, A., Taneja, N., and Sharma, M. (2012). Viability kinetics, induction, resuscitation and quantitative real-time polymerase chain reaction analyses of viable but non-culturable Vibrio cholerae $\mathrm{O} 1$ in freshwater microcosm. J. Appl. Microbiol. 112, 945-953. doi: 10.1111/j.1365-2672.2012.05255.x

Miyata, S. T., Kitaoka, M., Wieteska, L., Frech, C., Chen, N., and Pukatzki, S. (2010). The Vibrio cholerae type VI secretion system: evaluating its role in the human disease cholera. Front. Microbiol. 1:117. doi: 10.3389/fmicb.2010. 00117

Monira, S., Nakamura, S., Gotoh, K., Izutsu, K., Watanabe, H., Alam, N. H., et al. (2013). Metagenomic profile of gut microbiota in children during cholera and recovery. Gut Pathog. 5:1. doi: 10.1186/1757-4749-5-1

Mullins, J., Lukhnova, L., Aikimbayev, A., Pazilov, Y., Van Ert, M., and Blackburn, J. K. (2011). Ecological niche modeling of the Bacillus anthracis A1.a sub-lineage in Kazakhstan. BMC Ecol. 11:32. doi: 10.1186/1472-6785-11-32

Murtugudde, R. (2009). Regional earth system prediction: a decision-making tool for sustainability? Curr. Opin. Environ. Sustain. 1, 37-45. doi: 10.1016/j.cosust.2009.07.004

Peterson, A. T., and Robins, C. R. (2003). Using ecological-niche modeling to predict barred owl invasions with implications for spotted owl conservation. Conserv. Biol. 17, 1161-1165. doi: 10.1046/j.1523-1739.2003.02206.x

Preheim, S. P., Boucher, Y., Wildschutte, H., David, L. A., Veneziano, D., Alm, E. J., et al. (2011a). Metapopulation structure of Vibrionaceae among coastal marine invertebrates. Environ. Microbiol. 13, 265-275. doi: 10.1111/j.14622920.2010.02328.x

Preheim, S. P., Timberlake, S., and Polz, M. F. (2011b). Merging taxonomy with ecological population prediction in a case study of Vibrionaceae. Appl. Environ. Microbiol. 77, 7195-7206. doi: 10.1128/AEM.00665-11

Pruzzo, C., Vezzulli, L., and Colwell, R. R. (2008). Global impact of Vibrio cholerae interactions with chitin. Environ. Microbiol. 10, 1400-1410. doi: 10.1111/j.14622920.2007.01559.x

Senoh, M., Ghosh-Banerjee, J., Ramamurthy, T., Hamabata, T., Kurakawa, T., Takeda, M., et al. (2010). Conversion of viable but non-culturable Vibrio cholerae to the culturable state by co-culture with eukaryotic cells. Microbiol. Immunol. 54, 502-507. doi: 10.1111/j.1348-0421.2010.00245.x

Takeda, Y. (2011). Vibrio parahaemolyticus, enterotoxigenic Escherichia coli, enterohemorrhagic Escherichia coli and Vibrio cholerae. Proc. Jpn. Acad. Ser. B Phys. Biol. Sci. 87, 1-12. doi: 10.2183/pjab.87.1
Tamplin, M. L., Gauzens, A. L., Huq, A., Sack, D. A., and Colwell, R. R. (1990). Attachment of Vibrio cholerae serogroup $\mathrm{O} 1$ to zooplankton and phytoplankton of Bangladesh waters. Appl. Environ. Microbiol. 56, 1977-1980.

Telfer, S., Lambin, X., Birtles, R., Beldomenico, P., Burthe, S., Paterson, S., et al. (2010). Species interactions in a parasite community drive infection risk in a wildlife population. Science 330, 243-246. doi: 10.1126/science.1190333

Turner, J. W., Good, B., Cole, D., and Lipp, E. K. (2009). Plankton composition and environmental factors contribute to Vibrio seasonality. ISME J. 3, 1082-1092. doi: 10.1038/ismej.2009.50

Unterweger, D., Kitaoka, M., Miyata, S. T., Bachmann, V., Brooks, T. M., Moloney, J., et al. (2012). Constitutive type VI secretion system expression gives Vibrio cholerae intra- and interspecific competitive advantages. PLoS ONE 7:e48320. doi: 10.1371/journal.pone.0048320

Vandermeer, J. H. (1972). Niche theory. Annu. Rev. Ecol. Syst. 3, 107-132. doi: 10.1146/annurev.es.03.110172.000543

Waldor, M. K., and Mekalanos, J. J. (1996). Lysogenic conversion by a filamentous phage encoding cholera toxin. Science 272, 1910-1914. doi: 10.1126/science.272.5270.1910

Wei, Y., Ocampo, P., and Levin, B. R. (2010). An experimental study of the population and evolutionary dynamics of Vibrio cholerae $\mathrm{O} 1$ and the bacteriophage JSF4. Proc. R. Soc. B Biol. Sci. 277, 3247-3254. doi: 10.1098/rspb.2010.0651

World Health Organization. (2010). Cholera, Fact Sheet. Available at: http://www. who.int/mediacentre/factsheets/fs107/en/index.html (accessed June 10, 2013).

Zahid, M. S. H., Udden, S. M. N., Faruque, A. S. G., Calderwood, S. B., Mekalanos, J. J., and Faruque, S. M. (2008). Effect of phage on the infectivity of Vibrio cholerae and emergence of genetic variants. Infect. Immun. 76, 5266-5273. doi: 10.1128/IAI.00578-08

Conflict of Interest Statement: The authors declare that the research was conducted in the absence of any commercial or financial relationships that could be construed as a potential conflict of interest.

Received: 15 November 2013; paper pending published: 06 December 2013; accepted: 17 March 2014; published online: 02 April 2014.

Citation: Constantin de Magny G, Hasan NA and Roche B (2014) How community ecology can improve our understanding of cholera dynamics. Front. Microbiol. 5:137. doi: $10.3389 /$ fmicb.2014.00137

This article was submitted to Aquatic Microbiology, a section of the journal Frontiers in Microbiology.

Copyright (c) 2014 Constantin de Magny, Hasan and Roche. This is an open-access article distributed under the terms of the Creative Commons Attribution License (CC BY). The use, distribution or reproduction in other forums is permitted, provided the original author(s) or licensor are credited and that the original publication in this journal is cited, in accordance with accepted academic practice. No use, distribution or reproduction is permitted which does not comply with these terms. 